\title{
Comparison of immunohistochemistry, four in situ hybridization methods and quantitative polymerase chain reaction for the molecular diagnosis of HER2 status in gastric cancer: A study of 55 cases
}

\author{
LIBOR STANĚK ${ }^{1}$, TOMÁŠ ROZKOŠ ${ }^{2}$, JAN LACO $^{2}$, ALEŠ RYŠKA $^{2}$, LUBOŠ PETRUŽELKA $^{3}$, \\ MIROSLAV DŮRA ${ }^{4}$ and PAVEL DUNDR ${ }^{1}$ \\ ${ }^{1}$ Department of Pathology, First Faculty of Medicine, Charles University in Prague and General University Hospital in Prague, \\ CZ-128 00 Prague 2; ${ }^{2}$ The Fingerland Department of Pathology, Faculty of Medicine, Charles University in Prague \\ and University Hospital in Hradec Kralove, CZ-500 05 Hradec Králové; ${ }^{3}$ Department of Oncology, \\ First Faculty of Medicine, Charles University in Prague and General University Hospital in Prague, CZ-128 00 Prague 2; \\ ${ }^{4}$ First Faculty of Medicine, Charles University in Prague, CZ-128 00 Prague 2, Czech Republic
}

Received February 11, 2014; Accepted July 29, 2014

DOI: $10.3892 / \mathrm{mmr} .2014 .2530$

\begin{abstract}
In the current study, the sensitivity and specificity of methods of HER2 status detection were studied in 55 patients presenting with gastric/gastroesophageal junction carcinoma (30 intestinal and 25 diffuse), in small biopsy (endoscopy; $\mathrm{n}=33)$ and resection specimens $(\mathrm{n}=22)$. The primary objective of the present study was to compare various methods for the assessment of HER 2 status, with regards to the sensitivity and specificity of each method, as well as their concordance. In all cases, the status of HER2 was determined using immunohistochemistry (IHC), fluorescence in situ hybridization (FISH), silver in situ hybridization (SISH), and quantitative polymerase chain reaction (qPCR). The concordance rate between IHC and ISH was $100 \%$ for IHC 0 and $3+$. The concordance rate for IHC $1+$ was $100 \%$ between IHC and SISH, and $92.9 \%$ between IHC and FISH. The concordance rate among different FISH methods was $100 \%$, between FISH and SISH it was 96.2\%, and between qPCR and ISH methods it was $88.5 \%$. Thus, the results demonstrate that different in situ hybridization methods are comparable and that none were superior. Furthermore, the IHC and FISH methods were found to be comparable and the concordance rate was particularly good. qPCR analysis correlated well with the other methods and appears to be a possible alternative tool for detection of the HER 2 status. However, the
\end{abstract}

Correspondence to: Professor Pavel Dundr, Department of Pathology, First Faculty of Medicine, Charles University in Prague and General University Hospital in Prague, Studničkova 2, CZ-128 00 Prague 2, Czech Republic

E-mail:pdundr@seznam.cz

Key words: gastric cancer, HER2, immunohistochemistry, in situ hybridization, quantitative polymerase chain reaction concordance rate of qPCR with other methods was identified to be lower in the diffuse carcinoma group of endoscopy biopsy specimens; therefore investigation of further cases is required.

\section{Introduction}

HER2 proto-oncogene amplification and protein overexpression has been identified in $\sim 15-20 \%$ of patients with gastric and gastroesophageal adenocarcinoma worldwide (1-4). The Trastuzumab for Gastric Cancer (ToGA) trial revealed that trastuzumab, in combination with chemotherapy (platinum and fluoropyrimidine-based), significantly improves the overall survival of patients with HER2-positive advanced gastric cancer (GC) or gastroesophageal junction cancer (GJC) $(5,6)$. In 2010, based on the results of the ToGA study, the Food and Drug Administration granted approval of trastuzumab in combination with chemotherapy for the treatment of patients with GC/GJC. Subsequently, this therapy has been approved globally. However, the approach to HER2 testing in patients with GC and the optimal immunohistochemical and in situ hybridization methods remain a matter of discussion $(2,3,7,8)$. It is unclear whether there is a single method that is superior to others and thus, an optimal, in terms of the most sensitive and economically feasible, algorithm has not yet been established. Therefore, the present study compares various methods [immunohistochemistry (IHC), in situ hybridization (ISH), and quantitative polymerase chain reaction (qPCR)] in a series of GC/GJC specimens, focusing on the sensitivity and specificity of each method, as well as the level of their concordance.

\section{Patients and methods}

Patients. The present study included specimens from 55 patients with GC. This study was approved by the Ethics Committee of General University Hospital in Prague, Prague, Czech Republic. The study was designed as a methodological 
study rather than an epidemiological study. Additionally, the HER2 status was not compared in endoscopy and resection samples from the same patient. Thus, the samples were selected consecutively from the Department of Pathology, First Faculty of Medicine, Charles University in Prague and General Teaching Hospital in Prague, Prague, Czech Republic and The Fingerland Department of Pathology, Faculty of Medicine, Charles University in Prague and University Hospital in Hradec Králové, Hradec Králové, Czech Republic, according to the required criteria, which included the type of the tumor, the type of the sample and how representative the sample was with regard to the quantity of tumor tissue. In total, 22 resection specimens (15 intestinal carcinoma and seven diffuse carcinoma; male to female ratio, 10:12; age range, 33-89 years; mean age, 66.8 years) and 33 endoscopy biopsy specimens (15 intestinal carcinoma and 18 diffuse carcinoma; male to female ratio, 22:11; age range, 35-93 years; mean age, 66 years) were obtained.

Processing of the tissue. All specimens were immediately fixed in $10 \%$ formalin, routinely processed and embedded in paraffin. Histological evaluation was performed on slides, which were stained with hematoxylin and eosin, using an Olympus BX53 microscope (Tokyo, Japan).

IHC. IHC was performed using monoclonal rabbit antibody against the HER2 protein (clone 4B5, prediluted, Ventana anti-HER2/neu) according to the manufacturer's instructions. Automated HER2 staining was conducted by the immunostainer BenchMark Ultra following the manufacturer's instructions, with an ultraView Universal DAB Detection kit and Bluing reagent as the visualization reagent and chromogen. All materials were obtained from Roche Diagnostics GmbH, Mannheim, Germany.

The HER2 status for IHC was determined using the validated scoring system for GC (2). The samples with no reactivity or membranous reactivity in $<10 \%$ of tumor cells were scored as negative (0). Faint/barely perceptible membranous reactivity in $>10 \%$ of tumor cells (only in part of their membrane) was scored as negative (1+). Weak to moderate complete or basolateral membranous reactivity in $>10 \%$ of tumor cells was scored as equivocal (2+). Moderate to strong complete or basolateral membranous reactivity in $>10 \%$ of tumor cells was scored as positive (3+). In endoscopy biopsy specimens (not resection specimens), any cohesive $3+$ group of cells were scored as positive, irrespective of size (i.e. $<10 \%$ ).

Fluorescence ISH (FISH). Sections (thickness, 2-3 $\mu \mathrm{m}$ ) of paraffin-embedded tissue were processed for FISH using the following materials: i) PathVysion HER2 DNA Probe kit (Abbott Vysis, Downers Grove, IL, USA), ii) HER2 IQFISH pharmDx $^{\text {TM }}$ (Dako, Glostrup, Denmark) and iii) ZytoLight ${ }^{\circledR}$ SPEC HER2/CEN 17 Dual Color probe (ZytoVision GmbH, Bremerhaven, Germany). The assay procedures involving materials $\mathrm{i}$ and iii precisely followed the manufacturer's instructions.

Procedure I (PathVysion and ZytoLight). The slides were deparaffinized in xylene and pretreated in $0.2 \mathrm{~N} \mathrm{HCl}$ and subsequently in a NaSCN solution at $80^{\circ} \mathrm{C}$. The next step was the proteolytic treatment, and protease digestion was performed to obtain readable and conclusive FISH results. Protease II (25 mg; Abbott Vysis) in $50 \mathrm{ml}$ saline solution at $\mathrm{pH} 2.0$ was used with a digestion time of $45 \mathrm{~min}$ for the endoscopy biopsy specimens and $60 \mathrm{~min}$ for the resection specimens. The sections were then fixed in a buffered formalin solution. The FISH probe was added, the reaction was sealed with liquid rubber cement and the specimen and the DNA probe were co-denatured for $1 \mathrm{~min}$ at $85^{\circ} \mathrm{C}$ or $75^{\circ} \mathrm{C}$ for PathVysion or Zytolight, respectively, and hybridized at $37^{\circ} \mathrm{C}$ overnight in a ThermoBrite system (Abbott Vysis).

Procedure II (HER2 IQFISH). The slides were deparaffinized in xylene $(96 \%)$ and alcohol $(70 \%)$ for $2 \mathrm{~min}$, washed in buffer for $2 \mathrm{~min}$ and placed in a pretreatment solution at $95^{\circ} \mathrm{C}$ for $10 \mathrm{~min}$. After the pretreatment, the slides were washed twice in the buffer solution for 3 min each time, which was followed by a proteolytic treatment. Subsequently, the protease digestion was performed to obtain readable and conclusive FISH results [8-10 drops of pepsin (Dako Cytomotion) incubated at $37^{\circ} \mathrm{C}$ for $\left.8-10 \mathrm{~min}\right]$. The slides were washed twice more in the buffer solution ( $3 \mathrm{~min}$ each) and in alcohol at concentrations of 70, 85 and $96 \%$ (each for $2 \mathrm{~min}$ ). The FISH probe $(10 \mu \mathrm{l})$ was then applied, the reaction was sealed with liquid rubber cement, and the DNA probe and the specimen were co-denatured for $10 \mathrm{~min}$ at $66^{\circ} \mathrm{C}$ and then hybridized for $120 \mathrm{~min}$ in the ThermoBrite system at $45^{\circ} \mathrm{C}$. This was followed by washing twice in the buffer for $3 \mathrm{~min}$ each. The unbound probe was removed via stringent wash at room temperature and the slides were placed in a water bath at $63^{\circ} \mathrm{C}$ for $10 \mathrm{~min}$. Subsequently, the slides were dehydrated in 70,85 and $96 \%$ alcohol (each for $2 \mathrm{~min}$ ) and counterstained with 4',6-diamidino-2-phenylindol (DAPI).

For each sample a minimum of 20 non-overlapping tumor cell nuclei were evaluated according using an Olympus Provis AX70 fluorescence microscope at a magnification of x100 and the appropriate filter setting for detecting the presence of amplification signals. According to the ToGa trial criteria for HER2 amplification, a positive result was defined as a ratio of HER2:CEP17 $\geq 2$ (6).

Silver ISH (SISH). Automated SISH of HER2 was conducted using a Ventana Inform HER2 Dual ISH DNA Probe Cocktail assay according to the manufacturer's instructions. The SISH conditions for the BenchMark Ultra instrument were as follows: Treatment with cell conditioner CC2 twice for $32 \mathrm{~min}$ each, digestion with ISH protease III for $12 \mathrm{~min}$, hybridization for $6 \mathrm{~h}$, washing at $72^{\circ} \mathrm{C}$, silver staining with the ultraView SISH DNP Detection kit for $8 \mathrm{~min}$, red staining with the ultraView Red ISH DIG Detection kit for $8 \mathrm{~min}$, hematoxylin staining for $8 \mathrm{~min}$ and bluing staining with Bluing reagent for $4 \mathrm{~min}$. All materials were from Roche Diagnostics GmbH.The interpretation of Ventana SISH staining was the same as that applied to the manual FISH method.

$D N A$ isolation and $q P C R$ analysis. Deparaffinization of sections of formalin-fixed, paraffin-embedded tissue and the isolation of DNA was performed using standard procedures (9). The sections were deparaffinized in xylene and the 
DNA was extracted using a QIAamp DNA Mini kit (Qiagen, Hamburg, Germany).

HER2 amplification levels were quantified using a LightCycler 480 (Roche Diagnostics $\mathrm{GmbH}$ ) and a LightMix HER2/neu kit (Tib MolBiol GmbH, Berlin, Germany) according to the manufacturer's instructions. The kit provided a DNA calibrator to generate a calibration curve.

The PCR conditions were as follows: Initial denaturation for $10 \mathrm{~min}$ at $95^{\circ} \mathrm{C}$, followed by 50 cycles of denaturation for $10 \mathrm{sec}$ at $95^{\circ} \mathrm{C}$, annealing for $10 \mathrm{sec}$ at $60^{\circ} \mathrm{C}$ and extension for $10 \mathrm{sec}$ at $72^{\circ} \mathrm{C}$.

The level of HER2 DNA was normalized to the level of the housekeeping gene, ribosomal protein L23 (RPL23). qPCR amplification of each transcript was performed twice. For the purposes of the present study, the normalized ratio of HER2:RPL23 > 2 was considered to be positive for HER2 gene amplification (the cut-off was set according to the recommendations of a previous study) (10).

Data analysis. The concordance rate was calculated as the number of samples which produced positive results with $\geq 2$ methods. The threshold for positivity was set as $3+$ (positive) for immunohistochemistry, HER2:RPL23>2 for qPCR and HER2:CEP17 $\geq 2$ for ISH methods. The concordance was calculated among FISH methods, between FISH and SISH method, between ISH and IHC method ( $2+$ excluded), and between ISH and qPCR.

\section{Results}

Data obtained. From the 55 samples tested, 52 were examinable by all of the methods, including IHC, ISH and qPCR. All of the results are summarized in Table I.

$I S H$. Due to insufficient probe hybridization, certain samples could not be examined by HER2 IQFISH ( $n=3)$, ZytoLight SPEC HER2/CEN $17(n=2)$ and PathVysion HER-2 DNA $(n=1)$. Technical difficulties, including a weak hybridization signal and a high hybridization background, were encountered in nine samples tested by ZytoLight SPEC HER2/CEN 17, eight samples tested by HER2 IQFISH, and three samples tested by PathVysion HER-2 DNA. From the 52 samples which could be examined by all three FISH methods, there were nine amplified cases (three intestinal carcinomas from resection specimens, two intestinal carcinomas from endoscopy biopsy specimens and four diffuse carcinomas from endoscopy biopsy specimens). There was no case of apparent chromosome 17 polysomy. The concordance rate among the FISH methods was $100 \%$. All 55 samples were examined by SISH and the concordance rate between the FISH and SISH methods was $96.2 \%$ (50/52 cases) (the two discordant cases are described in 'IHC versus ISH').

IHC. Of the 55 specimens examined, eight samples were $3+$ (14.5\%), eight samples were $2+(14.5 \%), 14$ samples were $1+$ $(25.5 \%)$ and 25 samples were $0(45.5 \%)$. With regard to the intestinal carcinomas, $19 / 30$ specimens were 0 or $1+$ (11/15 resection specimens and 8/15 endoscopy biopsy specimens), 6/30 specimens were $2+(1 / 15$ resection specimens and $5 / 15$ endoscopy biopsy specimens) and 5/30 specimens were $3+(3 / 15$ resection specimens and $2 / 15$ endoscopy specimens). Of the diffuse carcinomas, 20/25 specimens were 0 or $1+(6 / 7$ resection specimens and 14/18 endoscopy biopsy specimens), 2/25 specimens were $2+$ (1/7 resection specimens and $1 / 18$ endoscopy biopsy specimens) and $3 / 25$ specimens were $3+(0 / 7$ resection specimens and $3 / 18$ endoscopy specimens).

$q P C R$. All 55 samples were successfully analyzed by qPCR. A normalized ratio of HER2:RPL23 > 2 was considered to be positive and was found in nine cases (three intestinal carcinomas from resection specimens, two intestinal carcinomas from endoscopy biopsy specimens and four diffuse carcinomas from endoscopy biopsy specimens).

IHC versus ISH. The concordance rate between IHC and ISH was $100 \%$ for IHC 0 and IHC 3+. The concordance rate for IHC $1+$ was $100 \%$ between IHC and SISH, and $92.9 \%$ between IHC and FISH (13/14 cases). The only discrepancy was in the IHC 1+ case, amplification was not observed with SISH and qPCR, however, amplification was observed with all three FISH methods. In the group of IHC $2+$ cases (8/55 cases), there was one case in which amplification by SISH was observed, however, all three FISH methods and qPCR did not demonstrate amplification.

ISH versus $q P C R$. The concordance rate between ISH and qPCR was $88.5 \%$ (46/52 cases). In total, there were 7 discordant cases ( 5 cases were concordant between all ISH methods, 2 cases were not). Six cases were in the diffuse carcinoma group (endoscopy biopsy specimens) and the seventh case was in the intestinal carcinoma group (endoscopy biopsy specimen). In three of the discordant cases, IHC and ISH were negative, however, amplification was observed in qPCR. In another two discordant cases, IHC and ISH were positive, however, amplification was not observed in qPCR. The sixth case was IHC, SISH and qPCR negative, however, all three FISH methods were positive. The seventh case was an IHC 2+ intestinal carcinoma (endoscopy biopsy specimen) with weak amplification (HER2:CEP17=2.1) observed in SISH and no amplification was observed in any of the FISH methods or qPCR.

\section{Discussion}

GC is one of the most common types of cancer worldwide (11). This type of tumor is commonly diagnosed in the advanced stage and the mortality rate is high (12). In addition to conventional therapy (chemotherapy and surgery), the monoclonal antibody against the HER2/neu receptor (trastuzumab) in combination with chemotherapy has been approved in several countries based on the result of the ToGA trial for treatment of HER2-positive advanced tumors (6). However, accurate assessment of HER2 status is necessary for correct implementation of this type of therapy. The status of HER2 may be determined using IHC and ISH, either FISH or chromogenic/colorimetric ISH (CISH).

Studies focusing on HER2 positivity in GC and GJC demonstrate a wide range of positivity (7-42\%; mean, $19 \%)(2,13,19)$. There are various possible explanations for this, 
Table I. Results of HER2 analysis of carcinoma specimens using IHC, ISH and qPCR.

A, Resection specimens.

\begin{tabular}{|c|c|c|c|c|c|c|}
\hline \multirow[b]{2}{*}{ Carcinoma } & \multicolumn{5}{|c|}{$\begin{array}{c}\text { ISH HER 2:CEP17 } \\
\text { ratio values }\end{array}$} & \multirow{2}{*}{$\begin{array}{c}\text { HER2:RPL23 } \\
\text { ratio values }\end{array}$} \\
\hline & IHC score & Ventana & PathVysion & IQFISH & ZytoLight & \\
\hline \multicolumn{7}{|c|}{ Intestinal, patient no. } \\
\hline 1 & 0 & 1.12 & 1.36 & 1.26 & 1.86 & 0.269 \\
\hline 2 & 0 & 1.33 & 1.43 & 1.26 & 1.21 & 0.569 \\
\hline 3 & 0 & 1.39 & 1.69 & 1.86 & 1.36 & 0.693 \\
\hline 4 & 0 & 1.29 & 1.26 & 1.16 & 1.03 & 0.085 \\
\hline 5 & 0 & 1.19 & 1.14 & 1.09 & 1.00 & 0.009 \\
\hline 6 & 0 & 1.44 & 1.84 & 1.67 & 1.58 & 0.963 \\
\hline 7 & 0 & 1.35 & 1.26 & 1.13 & 1.06 & 0.264 \\
\hline 8 & 0 & 1.44 & 1.16 & 1.11 & 1.10 & 0.036 \\
\hline 9 & 0 & 1.33 & 1.13 & 1.08 & 1.00 & 0.006 \\
\hline 10 & 1 & 1.14 & 1.02 & 1.00 & 1.00 & 0.008 \\
\hline 11 & 1 & 1.12 & 1.79 & 1.91 & 1.82 & 1.296 \\
\hline 12 & 2 & 1.34 & 1.29 & 1.68 & 1.49 & 1.369 \\
\hline 13 & 3 & 8.64 & 4.96 & 4.12 & 5.11 & 8.569 \\
\hline 14 & 3 & 8.85 & 6.45 & 4.92 & 3.99 & 5.590 \\
\hline 15 & 3 & 11.36 & 5.16 & 3.21 & 2.99 & 5.230 \\
\hline \multicolumn{7}{|c|}{ Diffuse, patient no. } \\
\hline 31 & 0 & 1.42 & 1.03 & 1.00 & 1.00 & 0.254 \\
\hline 32 & 0 & 1.66 & 1.26 & 1.12 & 1.00 & 0.023 \\
\hline 33 & 0 & 1.07 & 1.00 & $\mathrm{~N}$ & 1.00 & 0.056 \\
\hline 34 & 0 & 1.04 & $\mathrm{~N}$ & $\mathrm{~N}$ & $\mathrm{~N}$ & 0.006 \\
\hline 35 & 0 & 1.58 & 1.35 & 1.24 & 1.32 & 0.259 \\
\hline 36 & 0 & 1.44 & 1.28 & 1.19 & 1.26 & 0.532 \\
\hline 37 & 2 & 1.32 & 1.12 & 1.03 & 1.02 & 0.079 \\
\hline
\end{tabular}

B, Endoscopy biopsy specimens.

ISH HER2:CEP17

ratio values

\begin{tabular}{|c|c|c|c|c|c|c|}
\hline \multirow[b]{2}{*}{ Carcinoma } & & \\
\hline & IHC score & Ventana & PathVysion & IQFISH & ZytoLight & qPCR \\
\hline \multicolumn{7}{|c|}{ Intestinal, patient no. } \\
\hline 16 & 0 & 1.00 & 1.00 & 1.00 & 1.00 & 0.009 \\
\hline 17 & 1 & 1.05 & 1.00 & 1.00 & 1.00 & 0.236 \\
\hline 18 & 1 & 1.12 & 1.36 & 1.03 & 1.25 & 0.063 \\
\hline 19 & 1 & 1.05 & 1.12 & 1.00 & 1.06 & 0.023 \\
\hline 20 & 1 & 1.12 & 1.14 & 1.00 & 1.09 & 0.009 \\
\hline 21 & 1 & 1.21 & 1.39 & 1.12 & 1.28 & 0.029 \\
\hline 22 & 1 & 1.19 & 1.00 & 1.00 & 1.08 & 0.012 \\
\hline 23 & 1 & 1.11 & 1.09 & 1.00 & 1.02 & 0.563 \\
\hline 24 & 2 & 1.43 & 1.23 & 1.03 & 1.21 & 0.036 \\
\hline 25 & 2 & 2.10 & 1.69 & 1.59 & 1.86 & 1.863 \\
\hline 26 & 2 & 1.02 & 1.00 & 1.00 & 1.00 & 0.005 \\
\hline
\end{tabular}

IHC, immunohistochemistry; ISH, in situ hybridization; qPCR, quantitative polymerase chain reaction; Ventana, Ventana Inform HER2 Dual ISH DNA Probe Cocktail assay; PathVysion, PathVysion HER-2 DNA; IQFISH, HER2 IQFISH pharmDx ${ }^{\text {TM }}$; ZytoLight, ZytoLight ${ }^{\circledR}$ SPEC HER2/CEN 17; N, no signal. 
Table I. cont.

\begin{tabular}{|c|c|c|c|c|c|c|}
\hline \multirow[b]{2}{*}{ Carcinoma } & \multicolumn{5}{|c|}{$\begin{array}{l}\text { ISH HER2:CEP17 } \\
\text { ratio values }\end{array}$} & \multirow{2}{*}{$\begin{array}{c}\text { HER2:RPL23 } \\
\text { ratio values }\end{array}$} \\
\hline & IHC score & Ventana & PathVysion & IQFISH & ZytoLight & \\
\hline 27 & 2 & 1.28 & 1.00 & 1.00 & 1.00 & 0.008 \\
\hline 28 & 2 & 1.15 & 1.23 & 1.03 & 1.15 & 0.035 \\
\hline 29 & 3 & 7.93 & 5.96 & 3.98 & 4.23 & 8.367 \\
\hline 30 & 3 & 5.90 & 3.98 & 3.23 & 3.86 & 7.328 \\
\hline \multicolumn{7}{|c|}{ Diffuse, patient no. } \\
\hline 38 & 0 & 1.10 & 1.00 & 1.00 & 1.00 & 0.236 \\
\hline 39 & 0 & 0.99 & 1.02 & 1.00 & 1.12 & 0.036 \\
\hline 40 & 0 & 1.07 & 1.03 & 1.02 & 1.09 & 0.691 \\
\hline 41 & 0 & 1.03 & 1.00 & 1.00 & 1.00 & 0.393 \\
\hline 42 & 0 & 1.00 & 1.00 & 1.00 & 1.00 & 15.143 \\
\hline 43 & 0 & 1.34 & 1.53 & 1.12 & 1.36 & 2.043 \\
\hline 44 & 0 & 1.10 & 1.20 & 1.00 & 1.12 & 0.029 \\
\hline 45 & 0 & 0.89 & 1.08 & 1.01 & 1.00 & 9.629 \\
\hline 46 & 0 & 1.15 & 1.00 & 1.03 & 1.06 & 0.627 \\
\hline 47 & 1 & 1.07 & 1.00 & $\mathrm{~N}$ & $\mathrm{~N}$ & 0.061 \\
\hline 48 & 1 & 1.16 & 3.24 & 3.56 & 2.96 & 0.632 \\
\hline 49 & 1 & 1.13 & 1.09 & 1.00 & 1.12 & 0.010 \\
\hline 50 & 1 & 1.09 & 1.16 & 1.00 & 1.06 & 1.696 \\
\hline 51 & 1 & 1.13 & 1.21 & 1.02 & 1.16 & 0.028 \\
\hline 52 & 2 & 1.06 & 1.00 & 1.00 & 1.03 & 0.005 \\
\hline 53 & 3 & 4.18 & 3.89 & 2.98 & 4.05 & 0.401 \\
\hline 54 & 3 & 9.78 & 7.36 & 5.56 & 6.89 & 6.698 \\
\hline 55 & 3 & 6.06 & 5.98 & 3.42 & 5.63 & 0.008 \\
\hline
\end{tabular}

IHC, immunohistochemistry; ISH, in situ hybridization; qPCR, quantitative polymerase chain reaction; Ventana, Ventana Inform HER2 Dual ISH DNA Probe Cocktail assay; PathVysion, PathVysion HER-2 DNA; IQFISH, HER2 IQFISH pharmDx ${ }^{\text {TM }}$; ZytoLight, ZytoLight ${ }^{\circledR}$ SPEC HER2/CEN 17; N, no signal.

including the use of different IHC antibodies, different fixation protocols, subjectivity and interpretation by pathologists, and the different scoring systems used $(2,3)$. Therefore, an accurate testing system is required to enable the use of trastuzumab in a clinical setting. The algorithm used in the testing of HER2 status in breast cancer appears to be appropriate, relying primarily on IHC and using ISH in cases of weak positivity (IHC 2+). However, based on the differences between the results of $\mathrm{GC}$ and breast cancer testing and the difficulties regarding the assessment of HER2 status in patients with GC, it has been recommended to use IHC and ISH testing together for patients with advanced GC or GJC $(2,14)$. An alternative approach has been described, which combines HER2 IHC and dual color ISH (Dual ISH) on a single slide (15). There are few studies comparing the IHC and ISH methods and these studies show variable results, however, the concordance rate is usually high with a range of $86.9-99 \%(8,16-20)$. The concordance rate between the various ISH methods is also high, commonly reaching $99 \%(3,21,22)$. The concordance rate between IHC $3+$ and amplification of the gene assessed by ISH methods is particularly high, commonly $>90 \%(3,16)$. However, in certain studies, amplification of the gene was observed by
ISH methods in a number of IHC-negative cases (0 and 1+). A previous study focused on interlaboratory agreement on HER2 scoring of GC or GJC. In the study, nine laboratories assessed the HER2 status of 100 specimens by IHC and ISH (CISH or SISH). The results indicated good agreement for the HER 2 copy number as determined by ISH, and the authors proposed that this is the optimal method for assessment of GC/GJC cases. An IHC 3+ score strongly predicted a positive ISH result, although the agreement for all IHC scores was only moderate (14).

The results of the present study revealed that the concordance rate among the various detection methods was good, with the concordance rate among all FISH methods at $100 \%$, 96.2\% between the FISH and SISH methods, and $88.5 \%$ between qPCR and the ISH methods. Discrepancies between ISH methods and qPCR occurred only in the endoscopy biopsy specimens, predominantly in diffuse carcinoma, and may have been caused by the technical difficulties associated with the analysis of these types of sample.

HER2 positivity defined as IHC $3+$ and/or all ISH $\geq 2$ was observed in 10/55 of the tumors analyzed in the current study (18.2\%). Further, amplification of HER2 was observed 
in another 3 cases by qPCR method, which were both IHC and ISH negative This data is in agreement with the scientific literature on the subject $(2,3,23)$. However, the present study was not designed to be an epidemiological study, but rather a methodological study. Regarding the technical and economic aspects, the FISH method is not generally available. Furthermore, this method requires cytogenetic skills and is more time-consuming when compared with the SISH method. In addition, FISH is more expensive and requires the use of a fluorescence microscope. The advantage of SISH, in addition to the cost and technical aspects, is that it uses a bright-field technique. However, the FISH method continues to be regarded as the gold standard in the assessment of HER2 gene status. qPCR can be performed on paraffin-embedded tissue and is sensitive and relatively inexpensive, as demonstrated by two previous studies on GC $(24,25)$. However, it should be regarded as an alternative method of HER2 detection, as further investigation into its use is required.

In conclusion, the present study has demonstrated good concordance rates between IHC and various ISH methods. Furthermore, qPCR has been demonstrated to be a valuable tool for the evaluation of HER2 gene amplification. These results are similar to those of a previous extensive and multicentric study focusing on the comparison of various ISH methods and qPCR in breast cancer, as well as being comparable with a previous study on breast carcinoma $(10,26)$.

\section{Acknowledgements}

The present study was supported by the Charles University in Prague institutional support projects PRVOUK-P27/LF1/1 (Complex Oncological Programme) and PRVOUK P37/11 (New Approaches in the Diagnosis and Treatment of Lifestyle Diseases and Diseases Associated with Aging Population), and by the Bank of Biological Material project BBM LM2010004.

\section{References}

1. Gravalos C and Jimeno A: HER2 in gastric cancer: a new prognostic factor and a novel therapeutic target. Ann Oncol 19: $1523-1529,2008$.

2. Hofmann M, Stoss O, Shi D, et al: Assessment of a HER2 scoring system for gastric cancer: results from a validation study. Histopathology 52: 797-805, 2008.

3. Pala EE, Bayol U, Ozguzer A and Akman O: HER2 status in gastric cancer: a comparison of two novel in situ hybridization methods (IQ FISH and dual color SISH) and two immunohistochemistry methods (A0485 and HercepTest ${ }^{\mathrm{TM}}$ ). Pathol Res Pract 209: 548-554, 2013.

4. Watson S, Validire P, Cervera P, et al: Combined HER2 analysis of biopsies and surgical specimens to optimize detection of trastuzumab-eligible patients in eso-gastric adenocarcinoma: a GERCOR study. Ann Oncol 24: 3035-3039, 2013.

5. Bang YJ: Advances in the management of HER2-positive advanced gastric and gastroesophageal junction cancer. J Clin Gastroenterol 46: 637-648, 2012.

6. Bang YJ, Van Cutsem E, Feyereislova A, et al; ToGA Trial Investigators: Trastuzumab in combination with chemotherapy versus chemotherapy alone for treatment of HER2-positive advanced gastric or gastro-oesophageal junction cancer (ToGA) a phase 3, open-label, randomised controlled trial. Lancet 376: $687-697,2010$
7. Daum O, Skálová A, Rozkos T and Laco J: Predictive diagnosis of HER2 in gastric adenocarcinoma. Cesk Patol 47: 160-163, 2011 (In Czech).

8. Ooi A, Kobayashi M, Mai M and Nakanishi I: Amplification of c-erbB-2 in gastric cancer: detection in formalin-fixed, paraffin-embedded tissue by fluorescence in situ hybridization. Lab Invest 78: 345-351, 1998.

9. Man YG, Moinfar F, Bratthauer GL, et al: An improved method for DNA extraction from paraffin sections. Pathol Res Pract 197: 635-642, 2001.

10. Jacquemier J, Spyratos F, Esterni B, et al: SISH/CISH or qPCR as alternative techniques to FISH for determination of HER2 amplification status on breast tumors core needle biopsies: a multicenter experience based on 840 cases. BMC Cancer 13: 351 , 2013.

11. Fock KM: Review article: the epidemiology and prevention of gastric cancer. Aliment Pharmacol Ther 40: 250-260, 2014.

12. Sehdev A and Catenacci DV: Gastroesophageal cancer: focus on epidemiology, classification, and staging. Discov Med 16: 103-111, 2013.

13. Brien TP, Depowski PL, Sheehan CE, Ross JS and McKenna BJ: Prognostic factors in gastric cancer. Mod Pathol 11: 870-877, 1998.

14. Fox SB, Kumarasinghe MP, Armes JE, et al: Gastric HER2 Testing Study (GaTHER): an evaluation of gastric/gastroesophageal junction cancer testing accuracy in Australia. Am J Surg Pathol 36: 577-582, 2012.

15. Hirschmann A, Lamb TA, Marchal G, Padilla M and Diebold J: Simultaneous analysis of HER2 gene and protein on a single slide facilitates HER 2 testing of breast and gastric carcinomas. Am J Clin Pathol 138: 837-844, 2012.

16. Cho EY, Srivastava A, Park K, et al: Comparison of four immunohistochemical tests and FISH for measuring HER 2 expression in gastric carcinomas. Pathology 44: 216-220, 2012.

17. Kiyose S, Igarashi H, Nagura K, et al: Chromogenic in situ hybridization (CISH) to detect HER2 gene amplification in breast and gastric cancer: comparison with immunohistochemistry (IHC) and fluorescence in situ hybridization (FISH). Pathol Int 62: 728-734, 2012.

18. Lee S, de Boer WB, Fermoyle S, Platten M and Kumarasinghe MP: Human epidermal growth factor receptor 2 testing in gastric carcinoma: issues related to heterogeneity in biopsies and resections. Histopathology 59: 832-840, 2011.

19. Takehana T, Kunitomo K, Kono K, et al: Status of c-erbB-2 in gastric adenocarcinoma: a comparative study of immunohistochemistry, fluorescence in situ hybridization and enzyme-linked immuno-sorbent assay. Int J Cancer 98: 833-837, 2002.

20. Yano T, Doi T, Ohtsu A, et al: Comparison of HER2 gene amplification assessed by fluorescence in situ hybridization and HER2 protein expression assessed by immunohistochemistry in gastric cancer. Oncol Rep 15: 65-71, 2006.

21. Grin A, Brezden-Masley C, Bauer S and Streutker CJ: HER2 in situ hybridization in gastric and gastroesophageal adenocarcinoma: comparison of automated dual ISH to FISH. Appl Immunohistochem Mol Morphol 21: 561-566, 2013.

22. Yan B, Yau EX, Choo SN, et al: Dual-colour HER2/chromosome 17 chromogenic in situ hybridisation assay enables accurate assessment of HER2 genomic status in gastric cancer and has potential utility in HER2 testing of biopsy samples. J Clin Pathol 64: 880-883, 2011.

23. Marx AH, Tharun L, Muth J, et al: HER-2 amplification is highly homogenous in gastric cancer. Hum Pathol 40: 769-777, 2009.

24. García-García E, Gómez-Martin C, Angulo B, et al: Hybridization for human epidermal growth factor receptor 2 testing in gastric carcinoma: a comparison of fluorescence in-situ hybridization with a novel fully automated dual-colour silver in-situ hybridization method. Histopathology 59: 8-17, 2011.

25. Kim MA, Jung EJ, Lee HS, et al: Evaluation of $H E R-2$ gene status in gastric carcinoma using immunohistochemistry, fluorescence in situ hybridization, and real-time quantitative polymerase chain reaction. Hum pathol 38: 1386-1393, 2007.

26. Tvrdík D, Staněk L, Skálová H, Dundr P, Velenská Z and Povysil C: Comparison of the IHC, FISH, SISH and qPCR methods for the molecular diagnosis of breast cancer. Mol Med Rep 6: 439-443, 2012. 